\title{
Vaccination with OVA-bound nanoparticles encapsulating IL-7 inhibits the growth of OVA-expressing E.G7 tumor cells in vivo
}

\author{
HIROKO TOYOTA $^{1}$, NORIKO YANASE ${ }^{1}$, TAKAYUKI YOSHIMOTO ${ }^{2}$, MITSUNORI HARADA ${ }^{3}$, \\ YASUKI KATO $^{3}$ and JUNICHIRO MIZUGUCHI ${ }^{1}$ \\ ${ }^{1}$ Department of Immunology, Tokyo Medical University, Shinjuku-ku, Tokyo 160-8402; \\ ${ }^{2}$ Department of Immunoregulation, Institute of Medical Science, Tokyo Medical University, \\ Shinjuku-ku, Tokyo 160-8402; ${ }^{3}$ NanoCarrier Co. Ltd., Kashiwa-shi, Chiba 277-0871, Japan
}

Received July 22, 2014; Accepted September 19, 2014

DOI: $10.3892 /$ or.2014.3603

\begin{abstract}
Immunotherapy has gained special attention due to its specific effects on tumor cells and systemic action to block metastasis. We recently demonstrated that ovalbumin (OVA) conjugated to the surface of nanoparticles (NPs) (OVA-NPs) can manipulate humoral immune responses. In the present study, we aimed to ascertain whether vaccination with OVA-NPs entrapping IL-7 (OVA-NPs-IL-7) are able to induce antitumor immune responses in vivo. Pretreatment with a subcutaneous inoculation of OVA-NPs delayed the growth of thymic lymphoma cells expressing a model tumor antigen OVA (E.G7-OVA), and OVA-NPs-IL-7 substantially blocked the growth of E.G7-OVA tumor cells, although NPs-IL-7 alone had a meager effect, as assessed by the mean tumor size and the percentage of tumor-free mice. However, pretreatment with OVA-NPs-IL-7 failed to reduce the growth of parental thymic tumor cells, suggesting that the antitumor effect was antigen-specific. A tetramer assay revealed that vaccination with OVA-NPs-IL-7 tended to enhance the proportion of cytotoxic T cells (CTLs) specific for OVA. When the tumorfree mice inoculated with OVA-NPs-IL-7 plus EG.7 cells were rechallenged with E.G7-OVA cells, they demonstrated reduced growth compared with that in the control mice. Thus, a single subcutaneous injection of OVA-NPs-IL-7 into mice induced tumor-specific and also memory-like immune responses, resulting in regression of tumor cells. Antigens on NPs entrapping IL-7 would be a promising carrier to develop and enhance immune responses, including humoral and cellular immunity
\end{abstract}

Correspondence to: Professor Junichiro Mizuguchi, Department of Immunology, Tokyo Medical University, 6-1-1 Shinjuku, Shinjuku-ku, Tokyo 160-8402, Japan

E-mail:mizu@tokyo-med.ac.jp

Abbreviations: TAA, tumor-associated antigen; DCs, dendritic cells; CTLs, cyototoxic T cells; NPs, nanoparticles; OVA, ovalbumin

Key words: vaccination, nanoparticles, tumor antigen, E.G7-OVA tumor cells as well as a method of drug delivery to a specific target of interest.

\section{Introduction}

Although currently well-established treatments, including chemotherapy, radiotherapy and surgery, are initially effective, some tumors have inherent or acquired resistance to current treatments. Alternative strategies such as cancer immunotherapy have attracted special attention (1-3). Immunotherapy could specifically target tumor cells through de novo expression of tumor-associated antigens (TAAs). TAA-expressing tumor cells are efficiently phagocytosed by immature dendritic cells (DCs) (4), which mature to generate co-stimulatory molecules and/or cytokines under inflammatory conditions. The mature DCs present antigens along with costimulatory signals to $\mathrm{T}$ cells, resulting in activation of T cells. CD8-positive $\left(\mathrm{CD} 8^{+}\right)$ $\mathrm{T}$ cells specifically recognize and respond to TAA-expressing tumor cells in combination with $\mathrm{CD} 4^{+} \mathrm{T}$ cells or their derived cytokines, resulting in generation of cytotoxic $\mathrm{T}$ cells (CTLs) that specifically kill tumor cells systemically (2). CD8-mediated immunotherapy has had considerable success in selected patients (2), and development of novel therapeutic approaches and optimization of current treatments hold great promise in the field of antitumor immunotherapy capable of controlling tumor growth, while minimizing damage to host tissues.

To evaluate CD8-mediated control of tumor growth, we employed the ovalbumin (OVA)-expressing thymoma cell line E.G7-OVA as a model for immunotherapy where OVA is a model TAA (5). Cytokines such as IL-2, IL-12, IFN- $\gamma$ and IL-7 have been used for modulating antitumor immune responses with limited success $(6,7)$. IL-7, originally known as a functional molecule sharing a receptor component ( $\gamma \mathrm{c}$ chain), plays a crucial role in the maintenance of memory $\mathrm{T}$ cells as well as the survival of naïve $\mathrm{T}$ cells (8). Moreover, a recent study demonstrated that IL-7 enhances immune responses against tumors $(9,10)$.

Nanoparticles (NPs) have been recently used as carriers to manipulate immune responses as well as a specific system to deliver drugs to a target of interest $(3,11-13)$. The physical characteristics of NPs, including size, hydrophobicity, stability, 
antigen loading and antigen-release kinetics, modulate immune responses. Moreover, immune responses are further modified by the antigen dose and method of conjugation of antigens on the surface of or inside NPs (11). Our preparation of biodegradable NPs consisting of poly(ethylene glycol) (PEG)-poly (glutamic acid) block copolymers carrying chemotherapeutic agents was effective for targeting tumors in vivo (14). We recently demonstrated that a single subcutaneous injection of OVA conjugated on the surface of NPs (OVA-NPs) induced OVA-specific IgG responses with poor IgE synthesis (15). The present study examined whether OVA-NPs loaded with IL-7 (OVA-NPs-IL-7) elicited antitumor immune responses in vivo. Vaccination with a single injection of OVA-NPs-IL-7 prophylactically prevented the growth of E.G7-OVA tumors but not parental thymic lymphoma cells in vivo, probably through induction of $\mathrm{CD}^{+}$CTLs.

\section{Materials and methods}

Mice. Female C57BL/6 mice were purchased from the Jackson Laboratory. The mice were maintained at the animal facility of Tokyo Medical University and used for experiments at the age of 8 weeks. The Ethics Committee of Animal Experiments of the Tokyo Medical University approved the experiments.

Cell lines. EL4 thymoma cells (TIB-39) and E.G7-OVA cells (a derivative of EL4 cells expressing OVA, CRL-2113) were obtained from the American Type Culture Collection (Manassas, VA, USA). Cells were maintained in RPMI-1640 supplemented with $10 \%$ (v/v) fetal bovine serum, $50 \mu \mathrm{M}$ 2-mercaptoethanol, and $100 \mu \mathrm{g} / \mathrm{ml}$ kanamycin at $37^{\circ} \mathrm{C}$ in a humidified atmosphere of $5 \% \mathrm{CO}_{2}$.

Preparation of OVA-NPs loaded with IL-7. Two types of PEG-pGlu with a carbonyl carbon of an amino acid partially replaced by a benzyl or octyl group through an ester linkage were synthesized at NanoCarrier Co., Ltd. (Kashiwa, Japan). One had a maleimide group at the PEG terminus and benzyl groups in pGlu, and the other had a methoxy group at the PEG terminus and octyl groups in pGlu. OVA-bound polymeric micelles were prepared as previously described (16). The micelles were separated from unbound OVA by ultrafiltration. IL-7 was encapsulated into the micelles at different weight percentages of protein to polymer of 5,2 and $0.7 \%$ at $\mathrm{pH} 6.0$. The final solvent contained $10 \%(\mathrm{w} / \mathrm{v})$ sucrose and $20 \mathrm{mM}$ sodium phosphate buffer ( $\mathrm{pH}$ 6.0). The micelles were stored at $-80^{\circ} \mathrm{C}$ prior to use.

Prophylactic protocolfor tumor growth. Mice were subcutaneously immunized with OVA-NPs-IL-7 in the back. Seven days later, the mice were challenged with E.G7-OVA or parental EL-4 tumor cells ( $1 \times 10^{6}$ cells in $100 \mu \mathrm{l}$ PBS/mouse), followed by assays for tumor growth.

Tetramerassayfor splenocytes.Detection of OVA-specific CTLs was performed using an $\mathrm{H}-2 \mathrm{~K}^{\mathrm{b}} / \mathrm{OVA}$ Tetramer-SIINFEKL kit (MBL, Nagoya, Japan). Briefly, splenocytes from immunized mice were stained with anti-mouse CD8-FITC (KT15) (MBL), followed by an assay using a FACSCanto II flow cytometer (BD Biosciences) according to the manufacturer's instructions.

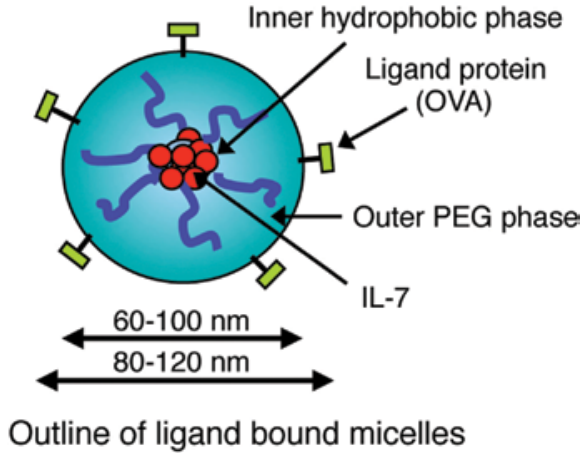

Figure 1. Preparation of OVA-NPs encapsulating IL-7. OVA-NPs were loaded with various concentrations of IL-7, which showed a size range of 80-100 nm in diameter. OVA, ovalbumin; NPs, nanoparticles.

Flow cytometric analysis. Single spleen cell suspensions from C57BL/6 mice primed with OVA-NPs-IL-7 and control mice were stained with anti-CD4-FITC, anti-CD62L-APC (BioLegend, San Diego, CA, USA), anti-CD8-FITC (MBL), and anti-CD44-PE/Cy7 (eBioscience, San Diego, CA, USA). Relative fluorescence intensities were measured using the FACSCanto II and analyzed with FACSDiva software (BD Biosciences).

Statistical analysis. Statistical analysis was performed with the Student's t-test. A p-value of $<0.05$ was considered to indicate a statistically significant result. Regression analysis was carried out using MS Excel software to determine the relationship between the two methods.

\section{Results}

Preparation of OVA-NPs loaded with IL-7. OVA was conjugated on the surface of NPs, and then IL-7 was loaded into the NPs (Fig. 1). The average diameter of the OVA-NPs-IL-7 was $100 \mathrm{~nm}$.

Pretreatment with OVA-NPS-IL-7 reduces the growth of E.G7-OVA tumor cells in vivo. Whether OVA on NPs in combination with IL-7 exert antitumor effects was examined in the present study. Pretreatment of C57BL/6 mice with OVA-NPs 7 days before E.G7-OVA tumor inoculation delayed tumor growth compared with the NPs alone (Fig. 2A). Vaccination of mice with OVA-NPs-IL-7 completely prevented the growth of E.G7-OVA tumor cells in 4 of the 5 mice for at least 28 days, although NPs-IL-7 had only a meager effect on tumor growth. However, the growth of parental EL4 tumor cells was almost comparable in the OVA-NPs-IL-7-treated and control NPs alone groups (Fig. 2B), suggesting that vaccination with OVA-NPs-IL-7 induced OVA-specific antitumor immunity in the mice.

OVA-NPs encapsulating various concentrations of IL-7 were examined for adjuvant action against tumor growth. OVA-NPs containing $5 \mu \mathrm{g}$ IL-7 completely blocked the growth of E.G7-OVA tumor cells in 4 of the 5 mice, whereas partial protection from tumor growth was obtained by 1.8 or $12.5 \mu \mathrm{g}$ IL-7 incorporated into the OVA-NPs (Fig. 3). Thus, $5 \mu \mathrm{g} \mathrm{IL-7}$ incorporated into the OVA-NPs was employed for the subsequent study. 

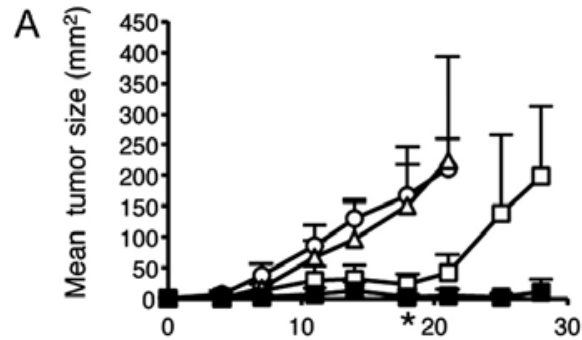

Days after tumor injection

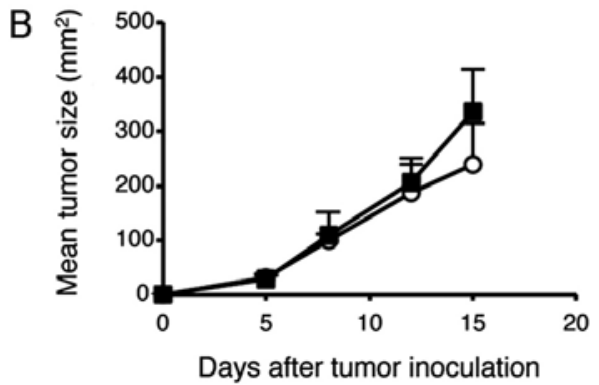

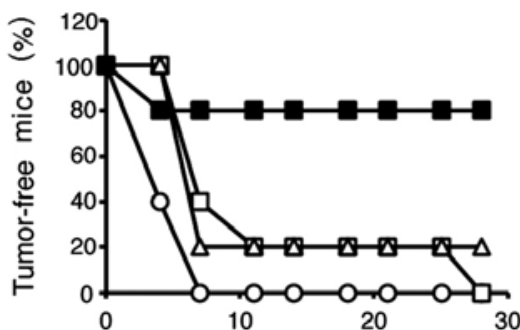

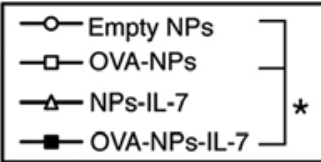

Days after tumor inoculation

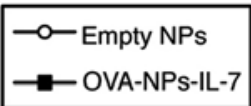

Figure 2. OVA-NPs-IL-7 blocks the growth of E.G7-OVA tumor cells in vivo. C57BL/6 mice were pretreated with OVA-NPs-IL-7, OVA-NPs, NPs-IL-7 and NPs alone, and then inoculated with $1 \times 10^{6}$ (A) E.G7-OVA or (B) EL4 tumor cells 7 days later, followed by assay every 3-4 days for tumor size up to (A) 28 and (B) 15 days, respectively. Data are means \pm SD for 5 mice/group. Data are from one of two independent experiments with essentially similar results. "p $<0.05$. OVA, ovalbumin; NPs, nanoparticles.

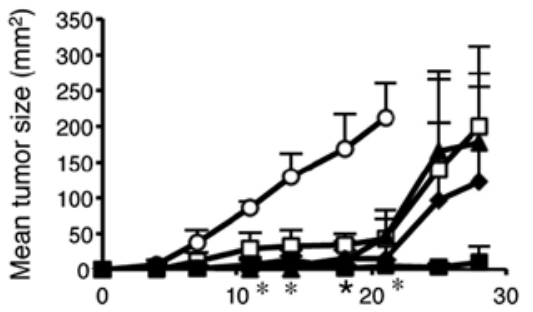

Days after tumor inoculation

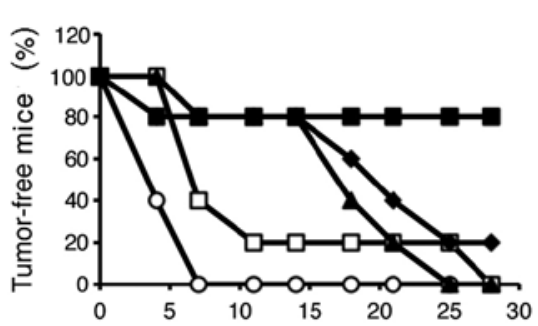

Days after tumor inoculation

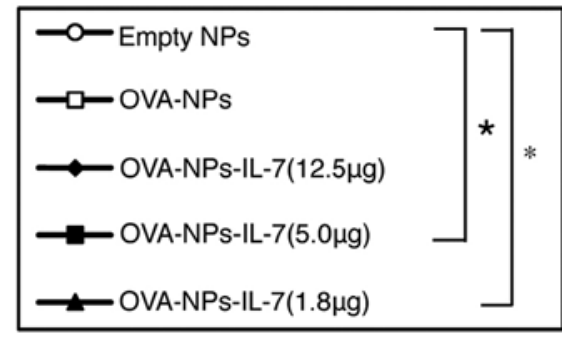

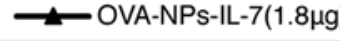

Figure 3. Dose response of IL-7 incorporated into OVA-NPs efficiently blocks the growth of E.G7-OVA tumor cells. Mice were primed with OVA-NPs containing various concentrations of IL-7, and then challenged with $1 \times 10^{6}$ E.G7-OVA tumor cells, followed by assay for tumor size. Data are means \pm SD for 5 mice/group. " $<<0.05$. OVA, ovalbumin; NPs, nanoparticles.

Generation of CTLs after vaccination with OVA-NPS-IL-7. Vaccination of tumor antigen with a suitable adjuvant induces tumor-specific CTLs, inhibiting tumor growth in vivo (11). Vaccination with OVA-NPs-IL-7 tended to enhance a portion of splenic CTLs specific for OVA compared with the control NPs alone (Fig. 4A). To examine the relationship between CTLs and tumor size, tumor size was measured (Fig. 4B). A regression plot of CTLs and tumor size is shown in Fig. 4C. The coefficient of determination, $\mathrm{R}^{2}=0.988$, suggested a good correlation between CTLs and tumor size. Thus, OVA-NPsIL-7-induced CTLs may have contributed to prevention of tumor growth in vivo.

Generation of memory-like T cells. Whether memory-like cells were generated by the immunization with OVA-NPs-IL-7 together with E.G7-OVA was examined. The tumor-free mice receiving OVA-NPs-IL-7 together with E.G7-OVA tumor cells showed partial resistance to rechallenge with E.G7-OVA cells (Fig. 5A), suggesting that this immunization generated a memory-like cell. Then, we analyzed $\mathrm{CD}^{+}$and $\mathrm{CD}^{+} \mathrm{T}$ cells from mice that had been immunized with OVA-NPs-IL-7 in combination withE.G7-OVA. The $\mathrm{CD} 4^{+} \mathrm{T}$ cells from the rechallenged mice expressed a memory phenotype $\left(\mathrm{CD} 44{ }^{\mathrm{hi}} \mathrm{CD} 62 \mathrm{~L}^{+}\right)$ relative to the controls (39.5 vs. 29.9) (Fig. 5B). The percentage of memory $\mathrm{CD}^{+} \mathrm{T}$ cells from the primed mice tended to be higher than that from the control mice, although statistically not significant. Thus, priming with OVA-NPs-IL-7 along with E.G7-OVA generated memory-like T cells in vivo, which may contribute to prevention of the growth of subsequently inoculated E.G7-OVA cells.

\section{Discussion}

Growth of tumors is controlled by the immune system (17). To induce a strong antitumor immune response to suppress tumor growth in vivo, a vaccine comprising a model tumor-associated antigen OVA on NPs loaded with IL-7 was prepared. A single immunization with OVA-NPs-IL-7 suppressed the growth of 

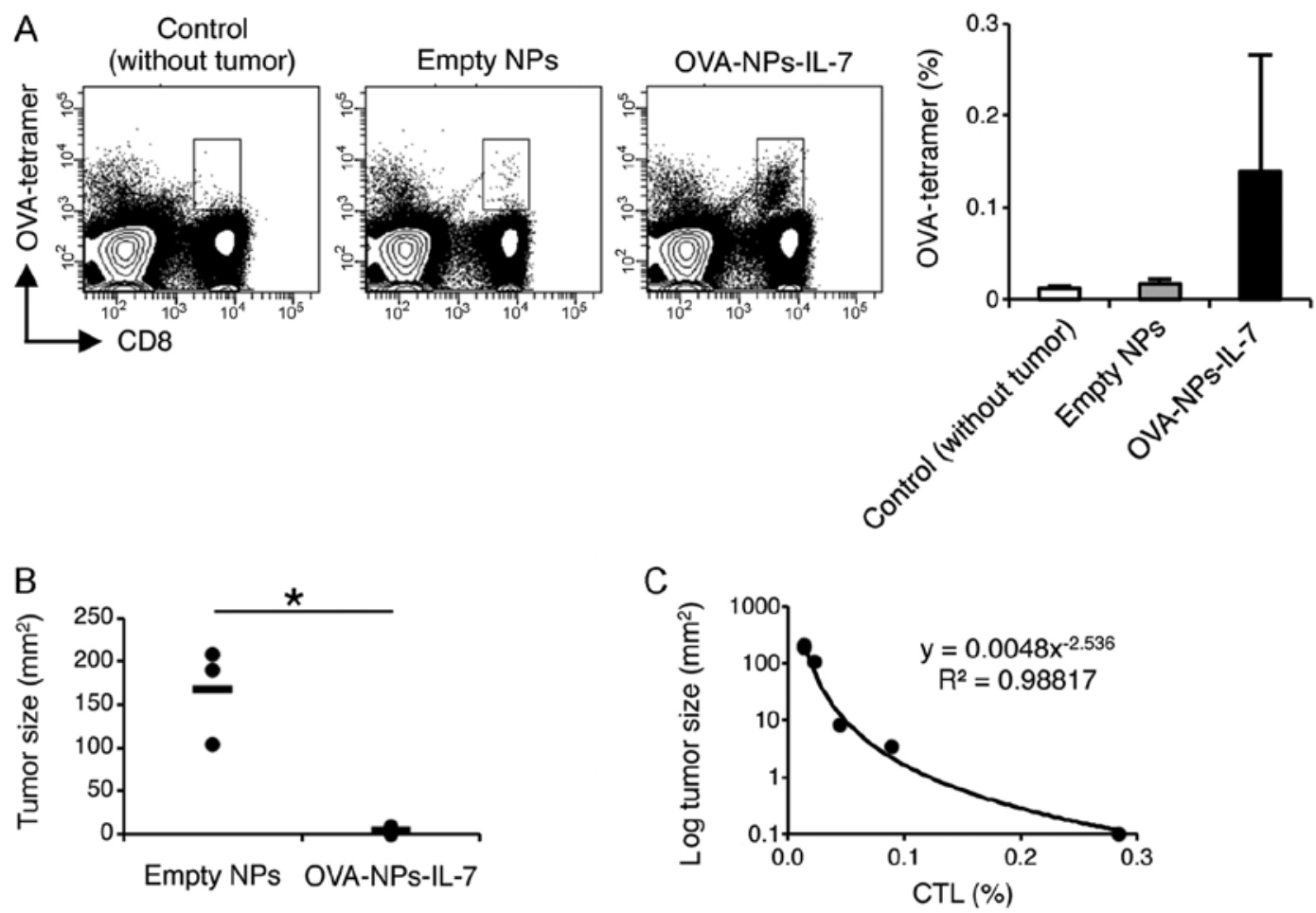

Figure 4. OVA-NPs-IL-7 generates OVA-specific CTLs in vivo. (A) Spleen T cells were prepared from mice primed with OVA-NPs-IL-7 or control NPs alone or in combination with E.G7 tumor inoculation 21 days previously, followed by assessment with tetramer assay ( 3 independent examples), as described in Materials and methods. (B) Tumor size of the mice was obtained when the spleen was removed. " $\mathrm{p}<0.05$. (C) Regression analysis was carried out to determine the relationship between tumor size and CTLs. OVA, ovalbumin; NPs, nanoparticles; CTL, cytotoxic T cell.

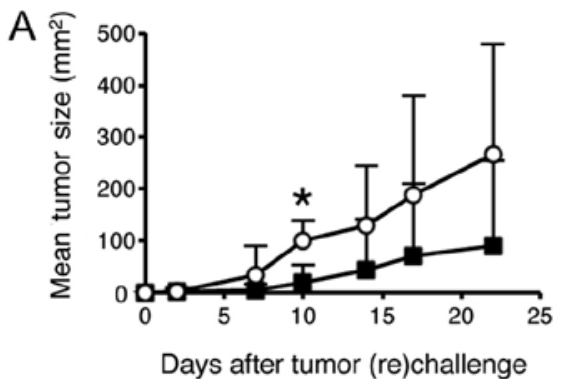

B

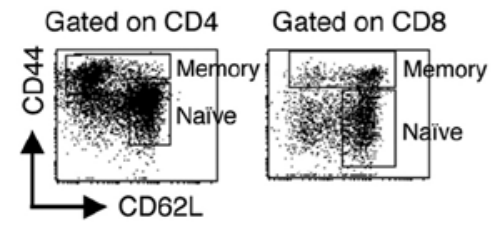

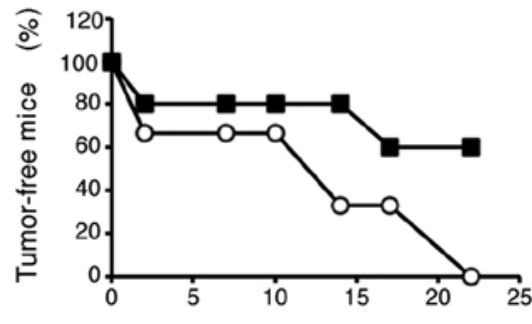

Days after tumor (re)challenge

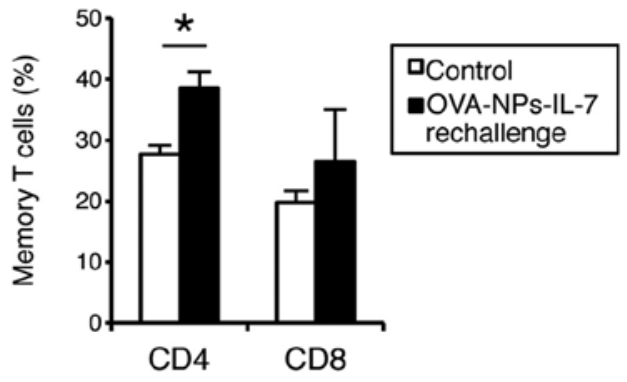

Figure 5. Single injection of OVA-NPs-IL-7 generates memory-like cells in vivo. (A) Tumor-free mice (n=5) receiving OVA-NPs-IL-7 together with E.G7-OVA tumor cells or control $(n=3)$ mice were then rechallenged with the same number of E.G7-OVA cells 53 days after the 1st inoculation, followed by assay for tumor size. Data are means \pm SD. ${ }^{*}$ p $<0.05$. (B) Spleen cells $(\mathrm{n}=3)$ from the E.G7-OVA rechallenged and control mice receiving E.G7 tumor cells alone were stained with anti-CD4-FITC, anti-CD8-FITC, anti-CD44-PE/Cy7 and anti-CD62L-APC 33 days after rechallenge. The percentage of T cells with a memory $\left(\mathrm{CD} 44^{\mathrm{hi}} \mathrm{CD} 62 \mathrm{~L}^{+}\right)$phenotype was calculated. ${ }^{\mathrm{p}}<0.05$. OVA, ovalbumin; NPs, nanoparticles.

OVA-expressing E.G7-OVA tumor cells. Memory-like CD4+ cells were generated after immunization with OVA-NPs-IL-7 in vivo, which may have contributed to the resistance to the rechallenge with E.G7-OVA cells. Thus, tumor antigen on NPs 
in the presence of cytokine IL-7 would function as a powerful prophylactic vaccine to prevent the growth of subsequent inoculations of tumor cells in vivo.

Synthetic particles mimicking the structure of microbes function as powerful immune adjuvant $(3,18)$. The adjuvant activity can be modified by physical characteristics of NPs including size, antigen loading and antigen-release kinetics. Our preparation of OVA-NPs-IL-7 $(\sim 100 \mathrm{~nm})$ prevented the growth of EG.7-OVA but not parental EL4 tumors (Fig. 2B). OVA-NPs may be endocytosed by DCs or macrophages to cross-prime $\mathrm{CD} 8^{+} \mathrm{T}$ cells in regional lymph nodes (19), since small particles in a range of $20-200 \mathrm{~nm}$ freely migrate to draining lymph nodes from the injection site (20). A slow release of IL-7 from OVA-NPs (13) would facilitate to activate and maintain $\mathrm{CD}^{+}$-memory $\mathrm{T}$ cells.

Cytokines produced by a variety of cells enhance or inhibit immune responses against pathogens and tumors. A clinical cancer trial demonstrated that IL-7 has efficacy as a therapeutic agent (21). IL-7 promotes the survival of effector $\mathrm{T}$ cells as well as memory $\mathrm{T}$ cells (22-24). Consistent with these findings, a single injection of OVA-NPs-IL-7 tended to enhance the proportion of CTLs specific for OVA relative to the control. The proportion of CD8 ${ }^{+}$CTLs appeared to correlate with poor tumor growth (Fig. 4B) $(17,25)$. Moreover, OVA-NPs-IL-7 in combination with E.G7-OVA cells enhanced the proportion of a $\mathrm{CD} 4^{+}$memory phenotype (Fig. 5B), which may have contributed to the resistance to the rechallenge with E.G7-OVA cells (Fig. 5A). However, the proportion of memory $B$ cell phenotype was comparable between OVA-NPs-IL-7 and the control group (Toyota et al, unpublished data). Thus, IL-7 appeared to shift the balance toward an antitumor effect by repressing regulatory networks (24), which are predominant in the tumor-bearing host $(26,27)$.

Collectively, we demonstrated that a single subcutaneous injection of OVA-NPs-IL-7 suppressed the growth in vivo of subsequently inoculated E.G7-OVA tumor cells expressing OVA, yet not parental EL4 cells. In addition to antitumor responses, OVA-NPs strongly generated humoral immunity including $\operatorname{IgG1}$ and IgG2a antibody production in mice (15). Thus, antigens conjugated on NPs would be a novel adjuvant enhancing cellular as well as humoral immune responses since they are stable, biodegradable and non-toxic to humans.

\section{References}

1. Sanlorenzo M, Vujic I, Posch C, et al: Melanoma immunotherapy. Cancer Biol Ther 15: 665-674, 2014.

2. Disis ML, Bernhard H and Jaffee EM: Use of tumour-responsive T cells as cancer treatment. Lancet 373: 673-683, 2009.

3. Yoo JW, Irvine DJ, Discher DE, et al: Bio-inspired, bioengineered and biomimetic drug delivery carriers. Nat Rev Drug Discov 10: 521-535, 2011.

4. Radford KJ, Tullett KM and Lahoud MH: Dendritic cells and cancer immunotherapy. Curr Opin Immunol 27: 26-32, 2014.

5. Moore MW, Carbone FR and Bevan MJ: Introduction of soluble protein into the class I pathway of antigen processing and presentation. Cell 54: 777-785, 1988.
6. Oppenheim JJ: Cytokines: Past, present, and future. Int J Hematol 74: 3-8, 2001.

7. Christian DA and Hunter CA: Particle-mediated delivery of cytokines for immunotherapy. Immunotherapy 4: 425-441, 2012.

8. Katzman SD, Hoyer KK, Dooms H, et al: Opposing functions of IL-2 and IL-7 in the regulation of immune responses. Cytokine 56: 116-121, 2011.

9. Liu S, Lizée G, Lou Y, et al: IL-21 synergizes with IL-7 to augment expansion and anti-tumor function of cytotoxic T cells. Int Immunol 19: 1213-1221, 2007.

10. Gou HF, Huang J, Shi HS, et al: Chemo-immunotherapy with oxaliplatin and interleukin-7 inhibits colon cancer metastasis in mice. PLoS One 9: e85789, 2014.

11. Oyewumi MO, Kumar A and Cui Z: Nano-microparticles as immune adjuvants: Correlating particle sizes and the resultant immune responses. Expert Rev Vaccines 9: 1095-1107, 2010.

12. Krishnamachari Y, Geary SM, Lemke CD, et al: Nanoparticle delivery systems in cancer vaccines. Pharm Res 28: 215-236, 2011.

13. Osada K, Christie RJ and Kataoka K: Polymeric micelles from poly(ethylene glycol)-poly(amino acid) block copolymer for drug and gene delivery. J R Soc Interface 6 (Suppl 3): S325-S339, 2009.

14. Matsumura Y and Kataoka K: Preclinical and clinical studies of anticancer agent-incorporating polymer micelles. Cancer Sci 100: 572-579, 2009.

15. Yanase N, Toyota H, Hata K, et al: OVA-bound nanoparticles induce OVA-specific IgG1, IgG2a, and IgG2b responses with low IgE synthesis. Vaccine 32: 5918-5924, 2014.

16. Harada M, Ohuchi M, Hayashi T and Kato Y: Prolonged circulation and in vivo efficacy of recombinant human granulocyte colony-stimulating factor encapsulated in polymeric micelles. J Control Release 156: 101-108, 2011.

17. DeNardo DG, Andreu P and Coussens LM: Interactions between lymphocytes and myeloid cells regulate pro- versus anti-tumor immunity. Cancer Metastasis Rev 29: 309-316, 2010.

18. Mahapatro A and Singh DK: Biodegradable nanoparticles are excellent vehicle for site directed in-vivo delivery of drugs and vaccines. J Nanobiotechnology 9: 55, 2011.

19. Andersen BM and Ohlfest JR: Increasing the efficacy of tumor cell vaccines by enhancing cross priming. Cancer Lett 325: 155-164, 2012.

20. Manolova V, Flace A, Bauer M, et al: Nanoparticles target distinct dendritic cell populations according to their size. Eur J Immunol 38: 1404-1413, 2008.

21. Möller P, Sun Y, Dorbic T, et al: Vaccination with IL-7 gene-modified autologous melanoma cells can enhance the antimelanoma lytic activity in peripheral blood of patients with a good clinical performance status: a clinical phase I study. Br J Cancer 77: 1907-1916, 1998.

22. Li J, Huston G and Swain SL: IL-7 promotes the transition of CD4 effectors to persistent memory cells. J Exp Med 198: 1807-1815, 2003.

23. Schluns KS, Kieper WC, Jameson SC and Lefrançois L: Interleukin-7 mediates the homeostasis of naïve and memory CD8 T cells in vivo. Nat Immunol 1: 426-432, 2000.

24. Rochman Y, Spolski R and Leonard WJ: New insights into the regulation of T cells by $\gamma_{c}$ family cytokines. Nat Rev Immunol 9: 480-490, 2009.

25. Hussein MR: Tumour-infiltrating lymphocytes and melanoma tumorigenesis: an insight. Br J Dermatol 153: 18-21, 2005.

26. Egeblad M, Nakasone ES and Werb Z: Tumors as organs: Complex tissues that interface with the entire organism. Dev Cell 18: 884-901, 2010.

27. de Visser KE, Eichten A and Coussens LM: Paradoxical roles of the immune system during cancer development. Nat Rev Cancer 6: 24-37, 2006. 